\title{
SOME MODERN NOTIONS IN THE RATIONAL TEACHING OF ELEMENTARY ALGEBRA.
}

By JAs. F. MiLlis, Shortridge High School, Indianapolis, Ind.

It is my intention, in this paper, to suggest a few of those things in a course in elementary algebra which should receive and, in some schools are now receiving, more attention than they have received in the past.

What things should receive emphasis in the teaching of elementary algebra will be determined mainly by the purpose of putting the subject into the school course. Why do we teach algebra to all high school pupils? Why should girls in the high school be made to take algebra for a year and a half? What good are they to get from it? Is the time spent in the study of algebra as it is now studied well spent by the great body of students who do not go to college?

I believe that we will agree that the following three things constitute the main purposes of putting algebra into the high school course:

1. To gain accuracy and speed in the fundamental algebraic processes and a knowledge of algebraic principles;

2. To develop the habit of right thinking;

3. Through the extension of the child's notions of number and number processes to gain a mastery of the world on the quantitative side.

Accuracy and speed in the fundamental processes are fundamental of course. They are essential. But they are the means and not the end. They have long been thought, apparently, to be the end itself. That seems to be a widespread notion even today; Now I claim that the teaching of algebraic processes and algebraic principles for the sake of those processes and principles alone is indefensible. The teacher whose sole ambition is to make the pupil ready to take an examination in algebraic processes is not teaching at all. Rather, he is teaching the subject instead of the child. Then, I repeat, accuracy in using the rules which are arbitrarily laid down for the child in the performance of algebraic processes is not all that is to be gotten from the subject; though this is necessary.

Accuracy is to be gained in several ways. (I) By familiarity with the symbolism of algebra. The pupil must know the exact 
meaning of every symbol used. He must know the meaning of general numbers, i. e., letters used to represent numbers. Here is where teachers have always failed in high school work. I shall touch upon this matter later. The pupil must know thoroughly the symbols of operation and the exact order in which they operate. That is, he must learn early how to evaluate an algebraic expression, or function. Too little time is usually spent upon this phase of algebra work. It should be carried throughout the entire algebra course. (2) Accuracy requires a thorough acquaintance with the elementary processes. This is evident. (3) Accuracy requires the formation of careful habits. (4) Accuracy requires long practice-not in solving difficult problems, but by reviews throughout the whole course. These reviews should be systematic, a part of every day's work.

The second purpose in teaching algebra to children, and in my mind the main purpose, is, as outlined above, to develop the habit of right thinking. This, it seems to me, is the main excuse for requiring girls to put a year and a half upon the subject in the high school. To develop within the child the power to reason from given facts to an accurate conclusion-that is the essential thing. It is but an exercise in logic. The teacher who does not have this in mind in the conduct of every recitation in elementary algebra is really not teaching. In getting algebra the pupil should also get a mental training that will make him more able to deal with the problems in other subjects. As Fitch says, in another connection, "we must teach so as to develop the searching and inquiring spirit, the love of truth, and the habit of accurate reasoning." It is evident that for most children the chief good to be obtained from the study of algebra is that power to think correctly that can be used in other studies. We should teach the child how to get from the known to the unknown without assistance.

Now, in all rational teaching of children the following pedagogical principles are involved:

(I) The concrete comes before the abstract; the particular comes before the general; the unknown must follow gradually from the known; processes should precede rules.

(2) The mind really develops only by its own activity.

Hence, in our algebra teaching. in the high school we should strengthen the foundations. We should move gradually from the 
known to the unknown. We should close the gap between arithmetic and algebra. The notions of number which the child possesses when he leaves arithmetic should be gradually extended. He should grow his own algebra. Teaching algebra by rule is not teaching at all. That is but preparing the student to pass examinations. The child should be led by many particular cases to develop the rules for himself. Rules thrust into the mind from the outside are soon forgotten; but the power which is obtained when the pupil develops his own rules, when he grows his own algebra, is more lasting. Although the reasoning in more advanced mathematics is mostly deductive, the reasoning of young children in beginning algebra must be inductive in nature.

The third purpose of putting algebra into the course of study is, broadly speaking, to gain a mastery of the world on the quantitative side. It is possible for the child to use his algebra in mastering other things. This brings us at once face to face with the question of correlation. How can algebra be made to overlap other studies? How can the child be taught to appreciate and use it in mastering other fields of work in and out of school? What should elementary algebra do in the way of preparing for other studies?

Now, the consideration of the foregoing purposes of teaching algebra in the high school and the consideration of the method of teaching it which the principles of pedagogy have demanded are now causing a revision and vitalizing of the subject. Textbooks are being revised. The tendency is to strengthen some phases of the work which heretofore have been carelessly treated and to place emphasis upon some new things. I shall point out briefly some of these.

In accordance with the principle that in education we must proceed gradually from the known to the unknown, we art trying to bridge the chasm which has long existed between arithmetic and algebra. We cannot bring arithmetic up; so we shove algebra down. We make the approach to algebra more gradual. As algebra was formerly taught, the child was suddenly told on the first page of the book that letters could be used to represent numbers, and he was immediately set to work adding them, subtracting them, etc. The result was that few pupils, when they had finished algebra, could tell the meaning of $a, m$, or $x$, or any other letter, when used to represent number. Now 
we should take care to teach the pupil the meaning of general, or literal, numbers and not be in such great haste to see them add and subtract. The rational way to do this is to make use of the pupil's present knowledge. Take such a principle as

$$
\text { distance }=\text { rate } \times \text { time, }
$$

which the pupil knows will apply in every particular problem in uniform motion, and show that by replacing the words "distance," "rate," and "time," in the statement of the principle by the first letters of the words, the principle may be expressed in symbols by

$$
d=r \times t
$$

Then show that here the letters stand for numbers which have different particular values in different particular problems.

Take the rule for getting the area of a rectangle, which says area $=$ base $X$ altitude,

and show that this may be expressed in symbols by

$$
A=b \times h \text {, }
$$

where $A, b$, and $h$ stand for numbers which have different particular values in different particular rectangles.

Numerous illustrations of this kind will lead to a clear notion of the meaning and advantage of general number. We should reach the general notion from many particular cases. It is wrong to allow the pupil to use symbols until he knows clearly what they stand for.

The notion of the significance of general number, as well as a working knowledge of other symbols such as exponents, coefficients, etc., is most thoroughly established by a certain method of checking work which the pupil should be required to use religiously in the first year's work. This checking process consists of substituting particular values for all general numbers which are involved in the identities that the student is continually establishing. For example, in the problem to multiply $a^{2}+a b$. $+b^{2}$ by $a^{2}-a b+b^{2}$, the pupil gets the product $a^{4}+a^{2} b^{2}+b^{4}$. In the multiplicand, the multiplier and the product let the pupill substitute 2 for $a$ and $\mathrm{I}$ for $b$. Then the multiplier becomes 3 , the mutiplicand 7 and the product $2 \mathbf{I}$, as it should. The work checks. Again he finds the factors of $x^{4}+x^{2} a^{3}-6 a^{0}$ to be $x^{2}$ $+3 a^{3}$ and $x^{2}-2 a^{3}$. By making $x=2$ and $a=\mathrm{I}$, the given expression becomes 14 , and the factors become 7 and 2 , respecttively, as they should. 
This checking process, which is a new feature of elementary algebra work, serves not only to make clear the meaning of all the symbols of elementary algebra, but it has other good educational values. It teaches the pupil to check his own work, and not to come to the teacher, or to an answer book, for assurance. Thus it makes the pupil independent, self-reliant, and accurate. These are foremost among the qualities which we are trying to develop in the pupil.

In connection with the pedagogical principle that we should proceed gradually from the known to the unknown, to my mind the most rational method of developing the idea of negative number should be the same as that used in developing other new kinds of numbers, such as fractions, surds and imaginaries. Treat negative number as the result of an indicated impossible subtraction, where the subtrahend is greater than the minuend. Thus, to subtract 7 from 3 , we have $3-7$, or breaking up the $7,3-3-4=0-4$, or -4 . Negative number is thus shown to be in nature a subtrahend. This notion of negative number is historically correct. This is how the human race got negative number in the first place, proceeding gradually from the known to the unknown, rather than reaching out into realms outside of algebra and suddenly dragging in the notion of opposites, and trying to make the child digest it.

Negative number, fractions, surds, and imaginary numberall of the new kinds of number have been developed by the same principle, the attempt to make the fundamental processes of numbers universal in their applications.

Another application of the law of pedagogy that the unknown should follow gradually from the known is found in the treatment of the fundamental laws of numbers, i. e., the laws of order, grouping, etc. The tendency to-day is to make these laws more prominent and their use more definite in developing the principles of elementary algebra. To attempt to prove these fundamental laws for the beginner is evidently foolish. Here the method of induction should be used. The pupil should be led by many particular examples to see that these laws hold in arithmetic and that they will hold equally well in algebra. Their application in developing the principles of algebra then becomes a simple matter. After the pwpil has become sufficiently mature to put his own irought into the work and has become familiar with algebraic 
language and algebraic processes-that is, in an advanced course in algebra-the rigorous proofs of these fundamental laws might well be undertaken. The method of induction is the only method for young children.

In line with the general movement to make the approach to algebra more gradual, to proceed gradually from the known to the unknown, to allow the pupil's mind to develop by its own activity, such difficult principles as are involved in the process of obtaining the H. C. F. by division should be postponed. H. C. F. and L. C. M. by factoring are sufficient for the purposes of elementary. algebra. Likewise, cube root by the formula, which is of no special practical use, the general theory of the quadratic, and some other subjects may well be postponed until the pupil is more mature. These difficult topics, which are the bugbear of most students, and which may easily be omitted from an elementary course, can be taken up later in connection with the review of the essentials as a part of the more advanced work.

In the resolution of equations pupils should not be allowed to use the term "transpose" until they have, by many particular problems, thoroughly mastered the meaning of the term. They should be required first to solve many equations by the use of axioms and finally be led to discover for themselves that the use of the axioms may be replaced by the more mechanical process of transposition. Aside from the pedagogical principle involved this method of teaching gives the pupil a clearer notion of the equation and its transformations.

The same facts apply in the case of the term "clear of fractions." In the beginning require the pupil to state each time what it is that he multiplies both members of the equation by, and do not allow him to get the erroneous idea that he multiplies one fraction by one quantity, another fraction by another quantity, etc.

One other point and I shall have finished. A number of things can be done in the way of correlating algebra and other subjects. Three of these things I shall mention briefly.

(I) Pupils have long been taught in algebra that the first letters of the alphabet represent known numbers and the last letters unknown numbers. It is only in algebra that this is done. In the algebraic problems of geometry and physics the unknown numbers are usually anything but $x, y$, and $z$. Here is where 
algebra fails to properly prepare for geometry and science work. To my mind, the best thing that can be done in algebra to prepare for these other studies is so to teach the algebra that when the pupil takes up the study of geometry and physics the algebra that he finds there will be familiar to him. That is practically the whole battle. Teach the pupil that any letter may represent the unknown number. Use $a, M, r, V^{\prime}$, and all kinds of letters as the unknown numbers in the equations which the pupil solves in the first year of his work. They are as good as $x$, and just as easy.. We may make use of the actual formulæ of geometry and physics as equations to be solved. Take such a formula as

$$
s=1 / 2 a t^{2},
$$

which expresses one of Newton's laws of motion. Solve it as a linear equation for $a$ and as a quadratic for $t$. So for the theorems of geometry. Take the theorem which gives the area of the surface of a sphere:

$$
\mathrm{S}=4 \pi r^{2}
$$

Solve this as a pure quadratic for $r$. Plenty of such equations can be had from physics and geometry. And they are as good for the purposes of algebra as any equations in $x, y$, and $z$. Evidently we do not have to teach geometry and physics to do this. Just familiarize the pupil with the algebraic language of these subjects.

(2) Most books give a chapter on variables and variation, then make no use of it. As an application of variation it has been found easy to show that equations may be used to express a law of science. Show that Newton's law of falling bodies can be expressed by the equation

$$
s=\mathrm{r} / 2 a t^{2},
$$

which shows that the distance, an object has fallen from rest varies directly as the square of the time. Such work in algebra would be good if it did nothing more than to show the pupil that algebra goes somewhere, that it has practical uses in the world.

(3) The graph is now taught in some high schools. By teaching the graph in elementary algebra a relation between algebra and geometry is established, and a foundation is laid for the use of the graph in science work. My own experience in teaching the graph in the high school has convinced me that it is certainly worth while. 